мерной группе // Изв. Сарат. ун-та. Нов. сер. Сер. Математика. Механика. Информатика. 2009. Т. 9, вып. 1. C. 14-19.
2012. Vol. 385. P. 1162-1178.

7. Лукомский С. Ф. О рядах Хаара на компактной нуль-

\title{
On Differential Operator in Compact Zero-dimensional Groups
}

\section{S. Kruss}

Saratov State University, 83, Astrakhanskaya str., Saratov, 410012, Russia, KrussUS@gmail.com

We define strong derivative on zero-dimensional compact group and find conditions under which the differential operator does not depend from an orthonormal system that defines this derivative. For multidimensional case we find conditions under which the differential operator does not depend from method of conversion multidimensional group in one-dimensional group. We obtain a clear view of annihilators in a multidimensional compact zero-dimensional group.

Key words: zero-dimensional groups, pseudo-differential operator, strong $\mathbf{P}$-adic derivative, strong $\mathbf{P}$-adic integral.

This work was supported by the Russian Foundation for Basic Research (project no. 13-01-00102).

\section{References}

1. Butzer P. L. Wagner H. J. Walsh - Fourier series and the concept of a derivative. Appl.Anal, 1973, vol. 3. no. 1, pp. 29-46.

2. Golubov B. I. A modified strong dyadic integral and derivative. Sbornik: Mathematics, 2002, vol. 193, no. 4, pp. 507-529. DOI: 10.4213/sm643.

3. Volosivets S. S. A modified $P$-adic integral and a modified $P$-adic derivative for functions defined on a halfaxis. Russian Math. [Izvestiya VUZ. Matematika], 2005. vol. 49, no. 6. pp. 25-36.

4. Kozyrev S. V. Wavelet theory as $p$-adic spectral

analysis. Izv. Math., 2002, vol. 66, no. 2, pp. 367-376. DOI: $10.4213 /$ im 381 .

5. Lukomskii S. F. Haar system on a product of zerodimensional compact group. Centr. Eur. J. Math., 2011, vol. 9, no. 3, pp. 627-639.

6. Lukomskii S. F. Multiresolution analysis on product of zero-dimensional Abelian groups. J. Math. Anal. Appl., 2012, vol. 385, pp. 1162-1178.

7. Lukomskii S. F. Haar series on compact zerodimmesional abelian group. Izv. Saratov Univ. (N.S.), Ser. Math. Mech. Inform., 2009, vol. 9, no. 1, pp. 14-19 (in Russian).

УДК 517.51

\section{ПРИБЛИЖЕНИЕ ФУНКЦИЙ В СИММЕТРИЧНЫХ И СВЯЗАННЫХ С НИМИ ГЕЛЬДЕРОВЫХ ПРОСТРАНСТВАХ ЛИНЕЙНЫМИ СРЕДНИМИ РЯДОВ ФУРЬЕ}

\section{Т. В. Лихачева}

Аспирантка кафредры теории фрункций и приближений, Саратовский государственный университет им. Н. Г. Чернышевского, lofinaT@mail.ru

В статье некоторые методы суммирования применяются к рядам Фурье-Виленкина в так называемых симметричных пространствах. Эти методы используют треугольные матрицы, суммы по строкам которых стремятся к нулю, с некоторыми ограничениями на разности коэфрфициентов. Тригонометрические аналоги наших результатов принадлежат М. Л. Митталу, Б. Э. Родесу, А. Гувену и др.

Ключевые слова: система Виленкина, линейные средние, пространство Гельдера, симметричное (перестановочно-инвариантное) пространство.

\section{1. ОСНОВНЫЕ ПОНЯТИЯ}

Пусть $\mathbf{P}=\left\{p_{n}\right\}_{n=1}^{\infty}-$ последовательность натуральных чисел такая, что $2 \leq p_{n} \leq N$ при $n \in \mathbb{N}$. Положим по определению $m_{0}=1, m_{n}=p_{1} \cdots p_{n}$ при $n \in \mathbb{N} ; \mathbb{Z}\left(p_{n}\right)=\left\{0,1, \cdots, p_{n}-1\right\}$. Каждое число $x \in[0,1)$ может быть представлено в виде

$$
x=\sum_{n=1}^{\infty} x_{n} / m_{n}, \quad x_{n} \in \mathbb{Z}\left(p_{n}\right) .
$$


Если $x=k / m_{j}, 0<k<m_{j}, k, j \in \mathbb{N}$, то мы берем разложение с конечным числом $x_{n} \neq 0$. Для $x, y \in[0,1)$ вида (1) положим $x \oplus y=z=\sum_{n=1}^{\infty} z_{n} / m_{n}, z_{n} \in \mathbb{Z} \cap\left[0, p_{n}\right), z_{n}=x_{n}+y_{n}\left(\bmod p_{n}\right)$. В случае, когда $z_{n}=p_{n}-1, n>n_{0}$, операция $x \oplus y$ не определена. Аналогично определяется обратная операция $x \ominus y$.

Каждое $k \in \mathbb{Z}_{+}$единственным образом представимо в виде

$$
k=\sum_{i=1}^{\infty} k_{i} m_{i-1}, \quad k_{i} \in \mathbb{Z}, \quad 0 \leq k_{i}<p_{i} .
$$

Для $x \in[0,1)$ и $k \in \mathbb{Z}_{+}$, записанных в виде $(1)$ и $(2)$, соответственно имеем:

$$
\chi_{k}(x)=\exp \left(2 \pi i \sum_{j=1}^{\infty} x_{j} k_{j} / p_{j}\right) .
$$

Система $\left\{\chi_{k}(x)\right\}_{k=0}^{\infty}$, называемая системой Виленкина, ортонормирована и полна в $L^{1}[0,1)$. Кроме того, при фиксированном $x \in[0,1)$ для почти всех $y \in[0,1)$ и всех $k \in \mathbb{Z}_{+}$имеют место равенства $\chi_{k}(x \oplus y)=\chi_{k}(x) \chi_{k}(y), \chi_{k}(x \ominus y)=\chi_{k}(x) \overline{\chi_{k}(y)}$. Эти свойства можно найти в [1, § 1.5].

Пусть $f \in L^{1}[0,1)$. Коэффициенты Фурье и частичная сумма Фурье $\left\{\chi_{k}(x)\right\}_{k=0}^{\infty}$ задаются формулами $\hat{f}(n)=\int_{0}^{1} f(t) \overline{\chi_{n}(t)} d t, n \in \mathbb{Z}_{+} ; S_{n}(f)(x)=\sum_{k=0}^{n-1} \hat{f}(k) \chi_{k}(x), n \in \mathbb{N}$. Сверткой функций $f, g \in L^{1}[0,1]$ называется $f * g(x)=\int_{0}^{1} f(x \ominus t) g(t) d t$. Справедливо равенство $S_{n}(f)(x)=f * D_{n}(x)$, где $D_{n}(x)=\sum_{k=0}^{n-1} \chi_{k}(x)$; для $\sigma_{n}(f)=\sum_{k=1}^{n} S_{k}(f) / n$ имеем: $\sigma_{n}(f)(x)=f * F_{n}(x)$, где $F_{n}(x)=\sum_{k=0}^{n-1} D_{k}(x) / n$, $n \in \mathbb{N}$.

Банахово пространство $E$ измеримых по Лебегу функций называется симметричным, если

1) из неравенства $|f(x)|<|g(x)|$ п. в. на $[0,1)$ и $g \in E$ следует, что $f \in E$ и $\|f\|_{E}<\|g\|_{E}$;

2) если $f$ и $g$ равноизмеримы и $g \in E$, то $f \in E$ и $\|f\|_{E}=\|g\|_{E}$.

Известно, что такое пространство $E$ вложено в $L^{1}[0,1)$ и что оператор растяжения $\left(\sigma_{\tau} f\right)(t):=$ $=f(t / \tau) X_{[0,1)}(t / \tau)$ непрерывен в $E$. Здесь $X_{\mu}-$ индикатор множества $\mu$. Пределы

$$
\alpha_{E}:=\lim _{\tau \rightarrow+0} \frac{\ln \left\|\sigma_{\tau}\right\|_{E \rightarrow E}}{\ln \tau}, \quad \beta_{E}:=\lim _{\tau \rightarrow \infty} \frac{\ln \left\|\sigma_{\tau}\right\|_{E \rightarrow E}}{\ln \tau}
$$

всегда существуют и называются соответственно верхним и нижним индексом Бойда пространства $E$. При этом всегда выполняется $0 \leq \alpha_{E} \leq \beta_{E} \leq 1$ [2, гл. 2]. В случае, когда $0<\alpha_{E} \leq \beta_{E}<1$, пространство $E$ обладает рядом хороших свойств, например, $\lim _{h \rightarrow 0}\|f(\cdot \ominus h)-f(\cdot)\|_{E}=0$ для любой $f \in E$ [3, с. 135]. Этот факт позволяет ввести модуль непрерывности и наилучшее приближение в $E$ формулами $\omega^{*}(f, \delta)_{E}=\sup _{0<h<\delta}\|f(\cdot \ominus h)-f(\cdot)\|_{E}, \delta \in(0,1), \mathscr{E}_{n}(f)_{E}:=\inf \left\{\left\|f-\sum_{i=0}^{n-1} a_{i} \chi_{i}\right\|_{E}, a_{i} \in \mathbb{C}\right\}$. Эти величины связаны неравенством типа А. В. Ефимова [3, формула $\left.\left(2^{\prime}\right)\right]$.

$$
2^{-1} \omega^{*}\left(f, 1 / m_{n}\right)_{E} \leq \mathscr{E}_{m_{n}} \leq\left\|f-S_{m_{n}}(f)\right\|_{E} \leq \omega^{*}\left(f, 1 / m_{n}\right)_{E}
$$

Кроме того, при $0<\alpha_{E} \leq \beta_{E}<1$ справедливо обобщенное неравенство Минковского:

$$
\left\|\int_{0}^{1} f(\cdot, y) d y\right\|_{E} \leq \int_{0}^{1}\|f(\cdot, y)\|_{E} d y
$$

[2, гл. 2, введение]. Некоторые другие свойства таких пространств $E$ установлены в леммах 3 и 4 и упомянуты в их доказательствах.

Если $\omega(t)$ возрастает и непрерывна на $[0,1], \omega(0)=0, \omega(t)>0$ при $t>0$, то будем обозначать как $\omega \in \Phi$. Функция $\omega \in \Phi$ принадлежит классу Бари - Стечкина $B_{1}$, если $\delta \int_{\delta}^{1} t^{-2} \omega(t) d t=O(\omega(\delta))$, 
$\delta \in(0,1)$. Будем говорить, что $\omega \in \Phi$ удовлетворяет $\Delta_{2}$-условию, если $\omega(2 t) \leq C \omega(t), t \in[0,1 / 2]$. По определению $H_{E}^{\omega}=\left\{f \in E: \omega^{*}(f, t)_{E} \leq C \omega(t)\right\}$, где $\omega \in \Phi$ и $C$ не зависит от $t \in[0,1]$.

Для $\omega \in \Phi$ пространство $H_{E}^{\omega}$ с нормой $\|f\|_{E, \omega}=\|f\|_{E}+\sup _{0<h<1} \omega^{*}(f, h)_{E} / \omega(h)$ является банаховым. Если $\omega$ удовлетворяет $\Delta_{2}$-условию, то выражение $\|f\|_{E, \omega}=\|f\|_{E}+\sup _{0<h<1}\|f(\cdot)-f(\cdot \ominus h)\|_{E} / \omega(h)$ задает эквивалентную норму в $H_{E}^{\omega}$.

Пусть $A=\left\{a_{n k}\right\}_{n, k=1}^{\infty}-$ нижнетреугольная матрица с неотрицательными элементами и $s_{n}(A)=\sum_{k=1}^{n} a_{n, k}$.

Обычно для треугольных матриц требуют выполнения условия $S_{n}(A)=1$, следуя $[4,5]$, мы требуем только $\lim _{n \rightarrow \infty} s_{n}(A)=1$. Введем А-преобразование ряда Фурье $f \in L^{1}[0,1)$ формулой $T_{n}(f)(x)=\sum_{k=1}^{\infty} a_{n, k} S_{k}(f)(x)$.

В работе [6] изучались оценки нормы $\left\|f-T_{n}(f)\right\|_{E}$ при $E=L^{p}[0,1)$ в терминах $\omega^{*}\left(f, a_{n, 0}\right)$ или $\omega^{*}\left(f, a_{n, n}\right)$. При этом рассматривались условия обобщенной монотонности $\left\{a_{n, k}\right\}_{n=1}^{n}$ типа RBVS [7]. Здесь изучаются другие условия на коэффициенты $\left\{a_{n, k}\right\}_{n=1}^{n}$, приспособленные к особенностям нормы пространства $E$, в частности к сходимости $S_{n}(f)$ к $f$ в $E$. Полученные результаты, как и в [7], применяются к приближениям в гельдеровых метриках.

\section{2. ВСПОМОГАТЕЛЬНЫЕ УТВЕРЖДЕНИЯ}

Функция $f(t)$ почти возрастает (почти убывает) на [a,b], если существует $K>0$ такое, что $f(u) \leq K f(v)(K f(u) \geq f(v))$ при $a \leq u \leq v \leq b$. Аналогично определяется почти возрастание (убывание) для последовательностей.

Лемма 1 [8]. Для $\omega \in \Phi$ следующие три условия равносильнь

$$
\begin{gathered}
\sum_{k=1}^{n} \omega(1 / k)=O(n \omega(1 / n)), \quad n \in \mathbb{N} ; \\
\delta \int_{\delta}^{1} t^{-2} \omega(t) d t=O(\omega(\delta)), \quad \delta \in(0,1) ; \\
\exists \alpha \in(0,1): t^{\alpha-1} \omega(t) \quad \text { почти убывает. }
\end{gathered}
$$

Замечание 1. Из условия $\left(S_{1}\right)$ следует, что $(2 t)^{\alpha-1} \omega(2 t) \leq K t^{\alpha-1} \omega(t)$, откуда следует $\omega(2 t) \leq$ $\leq 2^{\alpha-1} K \omega(t)$, т. е. выполнение $\Delta_{2}$-условия.

Лемма 2. Пусть $A=\left\{a_{n, k}\right\}_{n, k}^{\infty}$ - нижнетреугольная матрица с неотрицательными әлементами и $\omega \in B_{1}$. Если 1) $\left\{a_{n, k}\right\}_{k=1}^{n}$ почти убывает при всех $n \in \mathbb{N} u n a_{n, 1}=O(1), n \in \mathbb{N}$; или 2) $\left\{a_{n, k}\right\}_{k=1}^{n}$ почти возрастает при всех $n \in \mathbb{N}$ u na $a_{n,[n / 2]}=O(1)$; и в обоих случаях $s_{n}(A)=O(1)$ прu $n \in \mathbb{N}$, mo

$$
\sum_{k=1}^{n} a_{n k} \omega(1 / k)=O(\omega(1 / n)), \quad n \in \mathbb{N} .
$$

Доказательство. 1. В силу условия $\left(B_{1}\right)$ и неравенства $a_{n, k} \leq K a_{n, 1}$ при всех $k \in \mathbb{Z} \bigcap[1, n]$, получаем:

$$
\sum_{k=1}^{n} a_{n, k} \omega(1 / k) \leq K a_{n, 1} \sum_{k=1}^{n} \omega(1 / k)=O\left(n a_{n, 1} \omega(1 / n)\right)=O(\omega(1 / n)), \quad n \in \mathbb{N} .
$$

2. Согласно условию почти возрастания $\left\{a_{n, k}\right\}_{k=1}^{n}$ имеем: $a_{n, k} \leq K a_{n,[n / 2]}$ при всех $1 \leq k \leq[n / 2]$. Используя $\Delta_{2}$-условие для $\omega$ (см. замечание 1 ), ограниченность $s_{n}(A), \omega \in B_{1}$, находим, что

$$
\begin{aligned}
\sum_{k=1}^{n} a_{n k} \omega(1 / k) & =\left(\sum_{k=1}^{[n / 2]}+\sum_{k=[n / 2]+1}^{n}\right) a_{n, k} \omega(1 / k) \leq K a_{n,[n / 2]} \sum_{k=1}^{[n / 2]} \omega(1 / n)+ \\
+\omega(1 /[n / 2]) \sum_{k=[n / 2]+1}^{n} a_{n, k} & =O\left(n a_{n,[n / 2]+1} \omega(1 /[n / 2])\right)+O(\omega(1 /[n / 2])=O(\omega(1 / n)), \quad n \in \mathbb{N} .
\end{aligned}
$$

Лемма доказана. 
Будем писать что существует $f^{[1]} \in E$, если $f \in E$, и ряд $\sum_{k=1}^{\infty} k \hat{f}(k) \chi_{k}$ является рядом Фурье функции $f^{[1]}=g \in E$.

Лемма 3. Пусть $E-$ симметричное пространство, $0<\alpha_{E} \leq \beta_{E}<1$ и существует $f^{[1]} \in E$. Тогда $\left\|S_{n}(f)-\sigma_{n}(f)\right\|_{E}=O(1 / n), n \in \mathbb{N}$.

Доказательство. Легко видеть, что $\sigma_{n}(f)=\sum_{k=0}^{n-1}(1-k / n) \hat{f}(k) \chi_{k}$, поэтому

$$
\left\|S_{n}(f)-\sigma_{n}(f)\right\|_{E}=n^{-1}\left\|\sum_{k=1}^{n-1} k \hat{f}(k) \chi_{k}\right\|_{E}=n^{-1}\left\|S_{n}\left(f^{[1]}\right)\right\|_{E} .
$$

Согласно [9, теоремы 2.6.3, 2.3.11], если $0<1 / p_{2}<\alpha_{E} \leq \beta_{E}<1 / p_{1}<1$, то имеют место непрерывные вложения $L^{p_{2}}[0,1) \subset E \subset L^{p_{1}}[0,1)$ и Е является интеполяцонным между $L^{p_{1}}[0,1)$ и $L^{p_{2}}[0,1)$. В частности, из равномерной ограниченности операторов $S_{n}(f)$ на $L^{p_{1}}[0,1)$ и $L^{p_{2}}[0,1)$ $[10,11])$ следует равномерная ограниченность этих операторов на Е. Поэтому $\left\|S_{n}\left(f^{[1]}\right)\right\|_{E}=O(1)$, $n \in \mathbb{N}$, и из (5) следует утверждение леммы.

Лемма 4. Пусть $E-$ симметричное пространство, $0<\alpha_{E} \leq \beta_{E}<1, \omega \in \Phi$ удовлетворяет $\Delta_{2}$-условию, $f \in H_{E}^{\omega}$. Тогда

$$
\left\|f-S_{n}(f)\right\|_{E}=O(\omega(1 / n)), \quad n \in \mathbb{N} .
$$

Доказательство. Как установлено, при доказательстве леммы $3\left\|S_{n}(f)\right\|_{E} \leq C_{1}\|f\|_{E}$, откуда стандартным образом выводится неравенство $\left\|f-S_{n}(f)\right\|_{E} \leq\left(1+C_{1}\right) \mathscr{E}_{n}(f)_{E}$. В силу неравенства типа А. В. Ефимова (3), ограниченности $\left\{p_{n}\right\}_{n=1}^{\infty}$ и $\Delta_{2}$-условия на $\omega$ получаем при $m_{k} \leq n<m_{k+1}, k \in \mathbb{Z}_{+}$

$$
\mathscr{E}_{n}(f)_{E} \leq \mathscr{E}_{m_{k}}(f)_{E} \leq \omega^{*}\left(f, 1 / m_{k}\right)_{E} \leq C_{2} \omega\left(1 / m_{k}\right) \leq C_{3} \omega\left(1 / m_{k+1}\right) \leq C_{3} \omega(1 / n)
$$

для $n \in \mathbb{N}$. Из последнего легко вытекает утверждение леммы.

\section{3. ОСНОВНЫЕ РЕЗУЛЬТАТЫ}

Везде далее $E$ - симметричное пространство такое, что $0<\alpha_{E} \leq \beta_{E}<1, A-$ нижнетреугольная матрица с $a_{n, k} \geq 0$.

Теорема 1. Пусть $\omega \in B_{1}, f \in H_{E}^{\omega},\left|s_{n}(A)-1\right|=O(\omega(1 / n)), n \in \mathbb{N}$. Если А удовлетворяет условию 1) или условию 2) леммы 2, то

$$
\left\|f-T_{n}(f)\right\|_{E}=O(\omega(1 / n)) .
$$

Доказательство. По определению $T_{n}$ имеем:

$$
T_{n}(f)-f=\sum_{k=1}^{n} a_{n, k} S_{k}(f)-f=\sum_{k=1}^{n} a_{n, k}\left(S_{k}(f)-f\right)+\left(s_{n}(A)-1\right) f .
$$

Тогда согласно леммам 4 и 2 получаем

$$
\begin{aligned}
& \left\|T_{n}(f)-f\right\|_{E} \leq \sum_{k=1}^{n} a_{n, k}\left\|S_{k}(f)-f\right\|_{E}+\left|s_{n}(A)-1\right|\|f\|_{E}= \\
& =O\left(\sum_{k=1}^{n} a_{n, k} \omega(1 / k)+\omega(1 / n)\right)=O(\omega(1 / n)), \quad n \in \mathbb{N} .
\end{aligned}
$$

Теорема доказана.

Теорема 2. Пусть $\omega \in B_{1}, f \in H_{E}^{\omega}$ u $A$ такова, ито $\left|s_{n}(A)-1\right|=O(\omega(1 / n)), n \in \mathbb{N}$. Пусть также выполняется одно из условий:

1) $n \sum_{k=1}^{n}\left|a_{n, k}-a_{n, k+1}\right|=O(1)$,

2) $\sum_{k=1}^{n} k\left|a_{n, k}-a_{n, k+1}\right|=O(1),\left\{k\left|a_{n, k}-a_{n, k+1}\right|\right\}_{k=1}^{n}$ почти возрастает $u$

$$
n^{2}\left|a_{n,[n / 2]}-a_{n,[n / 2]+1}\right|=O(1),
$$


3) $\left\{k\left|a_{n, k}-a_{n, k+1}\right|\right\}_{k=1}^{n}$ почти убывает и $n^{2}\left|a_{n, 1}-a_{n, 2}\right|=O(1 / n), n \in \mathbb{N}$.

Тогда $\left\|f-T_{n}(f)\right\|_{E}=O(\omega(1 / n))$.

Доказательство. Снова по лемме 4 имеем:

$$
\left\|T_{n}(f)-f\right\|_{E} \leq \sum_{k=1}^{n} a_{n, k}\left\|S_{k}(f)-f\right\|_{E}+\left|s_{n}(A)-1\right|\|f\|_{E}=O\left(\sum_{k=1}^{n} a_{n, k} \omega(1 / k)+\omega(1 / n)\right) .
$$

В силу преобразования Абеля и условия $\left(B_{1}\right)$ получаем:

$$
\begin{aligned}
\left(\sum_{k=1}^{n} a_{n, k} \omega(1 / k)\right) & =O\left(\sum_{k=1}^{n-1}\left|a_{n, k}-a_{n, k+1}\right| \sum_{i=1}^{k} \omega(1 / i)+a_{n, n} \sum_{i=1}^{n} \omega(1 / i)\right)= \\
& =O\left(\sum_{k=1}^{n}\left|a_{n, k}-a_{n, k+1}\right| k \omega(1 / k)\right) .
\end{aligned}
$$

Здесь учтено, что $a_{n, n+1}=0$.

Из условия $\omega \in B_{1}$ и $\left(S_{1}\right)$ следует, что $C_{1} n^{1-\alpha} \omega(1 / n) \geq k^{1-\alpha} \omega(1 / k)$ при некотором $\alpha \in(0,1)$ и $n \geq k>1$, откуда вытекает неравенство $k \omega(1 / k) \leq C_{1}(k / n)^{\alpha} n \omega(1 / n) \leq C_{1} n \omega(1 / n)$. Поэтому при выполнении условия 1) правая часть (7) есть $O(\omega(1 / n))$ и, подставляя полученное соотношение в (6), получаем:

$$
\left\|T_{n}(f)-f\right\|_{E}=O(\omega(1 / n)), \quad n \in \mathbb{N} .
$$

В случаях 2) и 3) применяем лемму 2 к последовательности $\left\{k\left|a_{n, k}-a_{n, k+1}\right|\right\}_{k=1}^{n}$. Теорема доказана.

В качестве приложения теорем 1 и 2 рассмотрим средние Нерлунда-Вороного рядов ФурьеВиленкина. Пусть $\left\{q_{n}\right\}_{n=1}^{\infty}-$ последовательность положительных чисел, а элементы $A=\left\{a_{n, k}\right\}$ задаются формулой $a_{n, k}=q_{n-k+1}\left(\sum_{i=1}^{n} q_{i}\right)^{-1}, 1 \leq k \leq n$, и $a_{n, k}=0, k \geq n+1$ ([12, гл. 4]). Соответствующие А-суммы $T_{n}(f)$ обозначим через $N_{n}(f)$.

Следствие 1. Пусть $\omega \in B_{1}, f \in H_{E}^{\omega}$, и выполняется одно из условий

1) $\left\{q_{n}\right\}_{n=1}^{\infty}$ почти убывает $и n q_{n} \leq C \sum_{i=1}^{n} q_{i}, n \in \mathbb{N}$;

2) $\left\{q_{n}\right\}_{n=1}^{\infty}$ почти возрастает и $n q_{1} \leq C \sum_{i=1}^{n} q_{i}, n \in \mathbb{N}$;

3) $n \sum_{k=1}^{n-1}\left(\left|q_{k}-q_{k-1}\right|+q_{1}\right)=O\left(\sum_{k=1}^{n} q_{k}\right)$.

Тогда $\left\|N_{n}(f)-f\right\|_{E}=O(\omega(1 / n)), n \in \mathbb{N}$.

Доказательство. Заметим, что в данном случае $s_{n}(A)=1$ и что почти убывание (возрастание) $\left\{q_{n}\right\}_{n=1}^{\infty}$ соответствует почти возрастанию (убыванию) $\left\{a_{n, k}\right\}_{n=1}^{\infty}$. Поэтому достаточность условий 1) и 2) следует из теоремы 1 . Далее

$$
\sum_{k=1}^{n}\left|a_{n, k}-a_{n, k+1}\right|=\sum_{k=1}^{n-1}\left|a_{n, k}-a_{n, k+1}\right|+a_{n, n}=\left(\sum_{i=1}^{n} q_{i}\right)^{-1}\left(q_{1}+\sum_{i=1}^{n-1}\left|q_{n+1-k}-q_{n-k}\right|\right) .
$$

С помощью теоремы 2 устанавливаем достаточность условия 3). Следствие доказано.

В работах $[4,5]$ для приближения линейными средними тригонометрических рядов Фурье отдельно рассматривались $\omega(t)=t^{\alpha}, 0<\alpha<1$ и $\omega(t)=t$. При этом использовался факт, что $f \in \operatorname{Lip}(1, p)$, равносильно тому, что $f$ эквивалентна абсолютно непрерывной функции $f_{0}$ и $f_{0}^{\prime} \in L_{2 \pi}^{p}$ В нашем случае такой эквивалентности нет и мы используем класс $W^{1} E=\left\{f \in E: \exists f^{[1]} \in E\right\}$.

Теорема 3. Пусть $f \in W^{1} E,\left|s_{n}(A)-1\right|=O(1 / n)$, и выполнено одно из следующих трех условий:

$$
\begin{aligned}
& \text { 1) } n \sum_{k=1}^{n}\left|a_{n, k}-a_{n, k+1}\right|=O(1) \text {; } \\
& \text { 2) }\left\{a_{n, k}\right\}_{k=1}^{n} \text { yбывает nо } k \text { u na } a_{n, 1}=O(1) \text {; } \\
& \text { 3) }\left\{a_{n, k}\right\}_{k=1}^{n} \text { возрастает no } k \text {; для } n \in \mathbb{N} \text {. } \\
& \text { Тогда }\left\|f-T_{n}(f)\right\|_{E}=O(1 / n), n \in \mathbb{N} .
\end{aligned}
$$


Доказательство. Согласно лемме 5 из [13] для $f \in W^{1} E$

$$
\omega^{*}\left(f, 1 / m_{r}\right)_{E}=O\left(m_{r}^{-1} \omega^{*}\left(f^{[1]}, 1 / m_{r}\right)_{E}\right)=O\left(m_{r}^{-1}\right), \quad r \in \mathbb{N} .
$$

Отсюда в силу установленного при доказательстве леммы 4 неравенства $\left\|f-S_{n}(f)\right\|_{E} \leq C_{1} \mathscr{E}_{n}(f)_{E}$, неравенства типа А. В. Ефимова (3) и $\Delta_{2}$-условия для $\omega(t)=t$ легко следует $\left\|f-S_{n}(f)\right\|_{E}=O(1 / n)$, $n \in \mathbb{N}$.

Мы можем записать:

$$
\left\|T_{n}(f)-f\right\|_{E} \leq\left\|T_{n}(f)-S_{n}(f)\right\|_{E}+\left\|S_{n}(f)-f\right\|_{E}=\left\|T_{n}(f)-S_{n}(f)\right\|_{E}+O(1 / n) .
$$

Введем обозначение $A_{n, i}=\sum_{k=i+1}^{n} a_{n, k}$. С помощью преобразования Абеля находим, что

$$
\begin{gathered}
T_{n}(f)-S_{n}(f)=\sum_{k=1}^{n} a_{n, k} \sum_{i=0}^{k-1} \hat{f}(i) \chi_{i}-\sum_{i=0}^{n-1} \hat{f}(i) \chi_{i}= \\
=\sum_{i=0}^{n-1}\left(\sum_{k=i+1}^{n} a_{n, k}-\sum_{k=1}^{n} a_{n, k}\right) \hat{f}(i) \chi_{i}+\left(s_{n}(A)-1\right) S_{n}(f),
\end{gathered}
$$

откуда следует

$$
\begin{aligned}
& \left\|T_{n}(f)-S_{n}(f)\right\|_{E}=\left\|\sum_{i=1}^{n-1} i^{-1}\left(A_{n, i}-A_{n, 0}\right) i \hat{f}(i) \chi_{i}\right\|_{E}+O(1 / n)= \\
= & \left\|\sum_{i=1}^{n-2}\left(b_{n, i}-b_{n, i+1}\right) \sum_{j=1}^{i} j \hat{f}(j) \chi_{j}+b_{n, n-1} \sum_{j=1}^{n-1} j \hat{f}(j) \chi_{j}\right\|_{E}+O(1 / n),
\end{aligned}
$$

где $b_{n, i}=i^{-1}\left(A_{n, i}-A_{n, 0}\right)$. В силу условия $f^{[1]} \in E$ имеем: $\left\|\sum_{j=1}^{i} j \hat{f}(j) \chi_{j}\right\|_{E}=\left\|S_{i+1}\left(f^{[1]}\right)\right\|_{E}=O(1)$.

В то же время

$$
\begin{gathered}
\left|b_{n, i}-b_{n, i+1}\right|=\left|i^{-1}\left(A_{n, i}-A_{n, i+1}\right)+(i(i+1))^{-1}\left(A_{n, i+1}-A_{n, 0}\right)\right|= \\
=(i(i+1))^{-1}\left|(i+1) a_{n, i+1}-\sum_{j=1}^{i+1} a_{n, j}\right|=(i(i+1))^{-1}\left|\sum_{j=1}^{i} \sum_{k=j}^{i}\left(a_{n, k+1}-a_{n, k}\right)\right| \leq \\
\leq(i(i+1))^{-1} \sum_{k=1}^{i} \sum_{j=1}^{k}\left|a_{n, k+1}-a_{n, k}\right|=(i(i+1))^{-1} \sum_{k=1}^{i} k\left|a_{n, k+1}-a_{n, k}\right| .
\end{gathered}
$$

Аналогично

$$
b_{n, n-1}=(n-1)^{-1}\left|\sum_{k=1}^{n-1} a_{n, k}\right|=O(1 / n), \quad n \geq 2 .
$$

Подставляя (9) и последние оценки в (8), получаем в случае 1)

$$
\begin{gathered}
\left\|T_{n}(f)-S_{n}(f)\right\|_{E}=O\left(\sum_{i=1}^{n-2}(i(i+1))^{-1} \sum_{k=1}^{i} k\left|a_{n, k+1}-a_{n, k}\right|\right)+O(1 / n)= \\
=O\left(\sum_{k=1}^{n-2} \sum_{i=k}^{\infty}(i(i+1))^{-1} k\left|a_{n, k+1}-a_{n, k}\right|\right)+O(1 / n)=O\left(\sum_{k=1}^{n-2}\left|a_{n, k+1}-a_{n, k}\right|\right)+O(1 / n), \quad n \in \mathbb{N} .
\end{gathered}
$$

Если $\left\{a_{n, k}\right\}_{k=1}^{n}$ убывает или возрастает, то в силу второго равенства в (9) все $\left|b_{n, i}-b_{n, i+1}\right|$ раскрываются с одним знаком и $\sum_{i=1}^{n-2}\left|b_{n, i}-b_{n, i+1}\right|=\left|b_{n, 1}-b_{n, n-1}\right|$. 
При этом, если $\left\{a_{n, k}\right\}_{k=1}^{n}$ возрастает, то $a_{n, 1} \leq \sum_{k=1}^{n} a_{n, k} / n=O(1 / n), n \in \mathbb{N}$. Выше установлено, что $b_{n, n-1}=O(1 / n), n \geq 2$. Поскольку $\left|b_{n, 1}\right|=\left|A_{n, 1}-A_{n, 0}\right|=\left|a_{n, 1}\right|$, то из условий 2) и 3$)$ следует, что $\sum_{i=1}^{n-2}\left|b_{n, i}-b_{n, i+1}\right|=O(1 / n)$, откуда вытекает утверждение теоремы.

Замечание 2. Для $E=L^{p}, 1 \leq p \leq \infty, p_{i}=2$ и $T_{n}(f)=\sigma_{n}(f)$ известно, что $\left\|f-T_{n}(f)\right\|_{E} \neq o(1 / n)$ для функции, отличной от постоянной п.в. [14, теорема 2 (iii)]. Поэтому мы не рассматриваем $\omega(t)=o(t), t \rightarrow 0$.

Замечание 3. Теорема 1 является аналогом теоремы 1 из [5], теорема 2, по-видимому, не имеет тригонометрического аналога. Пункт 1) теоремы 3 является частичным аналогом теоремы 2 из [5], а пункты 2), 3) соответствуют пункту (ii) теоремы 4 в [4].

Теорема 4 является обобщением теоремы 8 из [7], где рассматривался случай $\omega(t)=t^{\alpha}, \mu(t)=t^{\beta}$, $0<\beta<\alpha$.

Теорема 4. Пусть $\omega \in \Phi$ удовлетворяет $\Delta_{2}$-условию, $\mu \in \Phi$, и при этом $\lambda(t)=\omega(t) / \mu(t)$ возрастаетна $(0,1)$ и $\lambda(0)=\lim _{t \rightarrow 0+0} \lambda(t)=0$. Тогда для $f \in H_{E}^{\omega}$ справедливо соотношение

$$
\left\|f-T_{n}(f)\right\|_{E, \mu}=O\left(\mu^{-1}(1 / n)\left\|f-T_{n}(f)\right\|_{E}+\lambda(1 / n)\left(1+\left\|T_{n}\right\|_{E \rightarrow E}\right)\right) .
$$

Доказательство. Поскольку $T_{n}(f)(x \ominus h)=T_{n}(f(\cdot \ominus h))(x)$, то при $h \geq 1 / n$ имеем:

$$
\begin{aligned}
\mu^{-1}(h) \| T_{n}(f)(x)-f(x)- & T_{n}(f)(x \ominus h)+f(x \ominus h)\left\|_{E} \leq 2 \mu^{-1}(h)\right\| T_{n}(f)-f \|_{E} \leq \\
& \leq 2 \mu^{-1}(1 / n)\left\|T_{n}(f)-f\right\|_{E} .
\end{aligned}
$$

Если же $0<h<1 / n$, то

$$
\left\|T_{n}(f)(\cdot)-f(\cdot)-T_{n}(f)(\cdot \ominus h)+f(\cdot \ominus h)\right\|_{E} \leq\left\|T_{n}(f(\cdot)-f(\cdot \ominus h))\right\|_{E}+\|f(\cdot)-f(\cdot \ominus h)\|_{E}
$$

и

$$
\begin{gathered}
\mu^{-1}(h)\left\|T_{n}(f)(\cdot)-f(\cdot)-T_{n}(f)(\cdot \ominus h)+f(\cdot \ominus h)\right\|_{E} \leq\left(1+\left\|T_{n}\right\|_{E \rightarrow E}\right) \omega(2 h) / \mu(h) \leq \\
\leq C_{1}\left(1+\left\|T_{n}\right\|_{E \rightarrow E}\right) \omega(h) / \mu(h) \leq C_{1}\left(1+\left\|T_{n}\right\|_{E \rightarrow E}\right) \lambda(h) \leq C_{1}\left(1+\left\|T_{n}\right\|_{E \rightarrow E}\right) \lambda(1 / n), \quad n \in \mathbb{N} .
\end{gathered}
$$

Объединяя оценки (10) и (11), получаем утверждение теоремы.

Следствие 2. Пусть соотношения $\omega \in B_{1}$, матрица удовлетворяет условиям теоремы 1 или теоремь 2, $\mu \in \Phi$ такова, ито $\lambda(t)=\omega(t) / \mu(t)$ возрастает на $(0,1) u \lambda(0)=\lim _{t \rightarrow 0+0} \lambda(t)=0$. Тогда для $f \in H_{E}^{\omega}$ имеет место соотношение

$$
\left\|f-T_{n}(f)\right\|_{E, \mu}=O(\lambda(1 / n)) .
$$

Доказательство. Из $\lim _{n \rightarrow \infty} s_{n}(A)=1$ следует, что $\left\|T_{n}(f)\right\|_{E} \leq s_{n}(A) \sup _{k}\left\|S_{k}(f)\right\|_{E}=O\left(\|f\|_{E}\right)$, т. е. $\left\|T_{n}\right\|_{E \rightarrow E}=O(1)$. Из теоремы 1 и 2 выводим оценку

$$
\mu^{-1}(1 / n)\left\|f-T_{n}(f)\right\|_{E}=O(\omega(1 / n) / \mu(1 / n))=O(\lambda(1 / n)) .
$$

Следствие доказано.

Следствие 3. Пусть $f \in W^{1} E$, матрица удовлетворяет условиям теоремы $3, \mu \in \Phi$ такова, что $t / \mu(t)$ возрастает на $(0,1)$ и $\lambda(0)=\lim _{t \rightarrow 0+0} \lambda(t)=0$. Тогда для $f \in H_{E}^{\omega}$ имеет место соотношение

$$
\left\|f-T_{n}(f)\right\|_{E, \mu}=O(\lambda(1 / n)), \quad n \in \mathbb{N} .
$$

\section{Библиографический список}

1. Голубов Б. И., Ефимов А. В., Сквориов В. А. Ряды и преобразования Уолша. Теория и применения. М. : Наука, 1987. 544 с.

2. Крейн С. Г., Петунин Ю. И, Семенов Е. М. Интер- поляция линейных операторов. М. : Наука, 1987. 400 с. 3. Volosivets S. S. On Hardy and Bellman transforms of series with respect to multiplicative systems in symmetric spaces // Analysis Math. 2009. Vol. 35, № 2. P. 131-148. 
4. Mittal M. L., Rhoades B. E., Mishra V. N., Singh $V$. Using infinite matrix to approximate functions of class $\operatorname{Lip}(\alpha, p)$ using trigonometric polynomials // J. Math. Anal. Appl. 2007. Vol. 326, № 1. P. 667-676.

5. Guven A. Trigonometric approximation in reflexive Orlicz spaces // Anal. Theory Appl. 2011. Vol. 27, № 2. P. 125-137.

6. Iofina T. V., Volosivets S. S. On the degree of approximation by means of Fourier - Vilenkin series in Holder and $L^{p}$ norm // East J. Approximations. 2009. Vol. 15, № 2. P. 143-158.

7. Leindler $L$. On the degree of approximation of continuous functions // Acta Math. Hungar. 2004. Vol. 104. P. 105-113.

8. Бари Н. К., Стечкин С. Б. Наилучшие приближения и дифференциальные свойства двух сопряженных функций // Тр. Моск. мат. о-ва. 1956. Т. 5. С. 488-522
9. Lindenstrauss J., Tzafriri L. Classical Banach spaces II. Berlin : Springer, 1973. 243 p.

10. Schipp $F$. On $L^{p}$-norm convergence of series with respect to product systems // Anal. Math. 1976. T. 2. C. 49-64.

11. Simon C. Verallgemeinerte Walsch-Fourierreihen // Acta Math. Hungar. 1976. Vol. 27, № 3-4. P. 329-341.

12. Харди Г. Расходящиеся ряды. М. : Изд-во иностр. лит., 1951. 504 с.

13. Zelin $H$. The derivatives and integrals of fractional order in Walsh-Fourier analysis with application to approximation theory // J. Approx. Theory. 1983. Vol. 39, № 3. P. 261-273.

14. Fridli $S$. On the rate of convergence of Cesaro means of Walsh-Fourier series // J. Approx. Theory. 1994. Vol. 76, № 1. P. 31-53.

\title{
Approximation of Functions in Symmetrical and Connected Holder Spaces by Linear Means of Fourier - Vilenkin Series
}

\section{T. V. Likhacheva}

\begin{abstract}
Saratov State University, 83, Astrakhanskaya str., Saratov, 410012, Russia, lofinaT@mail.ru
\end{abstract}
In this paper some summation methods are applied to Fourier-Vilenkin series in so called symmetric spaces. These methods use triangular matrix with sums in rows tending to zero and with some conditions on difference of coefficients. The triginometric counterpart of our results are due to M. L. Mittal, B. E. Rhoades, A. Guven, etc.

Key words: Vilenkin system, symmetrical (rearrangament invariant) space, Hölder space, linear mean.

\section{References}

1. Golubov B., Efimov A., Skvortsov V. Walsh series and transforms. Dodrecht; Boston; London, Kluwer Academic Publishers, 1991, 368 p. (Rus. ed. : Golubov B. I., Efimov A. V., Skvortsov V. A. Riady i preobrazovaniia Uolsha. Teoriia i primeneniia. Moscow, Nauka, 1987, 544 p.)

2. Krein S., Petunin J., Semenov E. Interpolation of linear operators. Translations Math. Monographs, vol. 55, Providene, R. I., Amer. Math. Soc., 1992. (Rus. ed. : Krein S. G., Petunin Iu. I, Semenov E. M. Interpoliatsiia lineinykh operatorov. Moskow, Nauka, 1987, 400 p.)

3. Volosivets S. S. On Hardy and Bellman transforms of series with respect to multiplicative systems in symmetric spaces. Analysis Math, 2009, vol. 35, no. 2, pp. 131-148. 4. Mittal M. L., Rhoades B. E., Mishra V. N., Singh V. Using infinite matrix to approximate functions of class Lip $(\alpha, p)$ using trigonometric polynomials. J. Math. Anal. Appl., 2007, vol. 326, no. 1, pp. 667-676.

5. Guven A. Trigonometric approximation in reflexive Orlicz spaces. Anal. Theory Appl., 2011, vol. 27, no. 2, pp. $125-137$.

6. Iofina T. V., Volosivets S. S. On the degree of approximation by means of Fourier-Vilenkin series in Holder and $L^{p}$ norm. East J. Approximations, 2009, vol. 15. no. 2. pp. 143-158.

7. Leindler L. On the degree of approximation of continuous functions. Acta Math. Hungar., 2004, vol. 104, pp. 105-113.

8. Bari N. K., Stechkin S. B. Nailuchshie priblizheniia i differentsial'nye svoistva dvukh sopriazhennykh funktsii. [Best approximations and differential properties of two conjugate functions]. Trudy Mosk. mat. obshchestra, 1956, vol. 5, pp. 488-522 (in Russian).

9. Lindenstrauss J., Tzafriri L. Classical Banach spaces II. Berlin, Springer, 1973, 243 p.

10. Schipp F. On $L^{p}$-norm convergence of series with respect to product systems. Anal. Math., 1976, vol. 2, pp. 49-64.

11. Simon P. Verallgemeinerte Walsch-Fourierreihen. Acta Math. Hungar., 1976, vol. 27, no. 3-4, pp. 329-341. 12. Hardy G. H. Divergent Series. New York, Oxford Univ. Press, 1949, 395 p. (Rus. ed. : Hardy G. Raskhodiashchiesia riady. Moscow, Izd-vo inostr. literatury, 1951, 505 p.)

13. Zelin $\mathrm{H}$. The derivatives and integrals of fractional order in Walsh-Fourier analysis with application to approximation theory. J. Approx. Theory., 1983, vol 39, no. 3, pp. 261-273.

14. Fridli S. On the rate of convergence of Cesaro means of Walsh - Fourier series. J. Approx. Theory., 1994, vol. 76 , no. 1 , pp. 31-53. 